regarding WHO recommended intake for fruit and vegetables. Cost, satiety, convenience and taste were the most important determinants of food choice. While sustainable development was never considered, health and nutrition were only secondary. Healthy eating was interpreted as balanced diet, although students admitted not adhering to balanced meals. Term time food behaviour exhibited by students included breakfast skipping; snacking on pastries and sugar-sweetened beverages (SSBs); low fruit and vegetable intake; late night eating; and eating out. The map of the University food environment showed a limited proportion of food outlets offering healthy food options. Compared to SSBs and pastries, fruit and vegetable outlets were particularly limited and farther from most student accommodation and lecture halls.

\section{P31 ABSTRACT WITHDRAWN}

\section{P32 IS ADOLESCENT MULTIPLE RISK BEHAVIOUR ASSOCIATED WITH REDUCED SOCIOECONOMIC STATUS IN YOUNG ADULTHOOD AND DO THOSE WITH LOW SOCIOECONOMIC BACKGROUNDS EXPERIENCE GREATER NEGATIVE IMPACT? FINDINGS FROM TWO UK BIRTH COHORTS}

L Tinner*, C Wright, J Heron, D Caldwell, R Campbell, M Hickman. Population Health Sciences, Bristol Medical School, University of Bristol, Bristol, UK

\subsection{6/jech-2020-SSMabstracts. 126}

Background Multiple risk behaviour (MRB) refers to the occurrence of two or more risk behaviours. Adolescent MRB is associated with multiple negative health outcomes including premature mortality and morbidity. It is also associated with deleterious social and educational outcomes such as reduced GCSE score, unemployment and police arrests. To date no studies have determined the relationship between adolescent MRB and young adult socioeconomic status (SES). The aim of the study was to examine the nature of the association between adolescent MRB and young adult SES including whether those with low early life SES experience greater negative impact.

Methods Two birth cohort studies; The British Cohort Study 1970 (BCS70) and The Avon Longitudinal Study of Parents and Children (ALSPAC), born in 1991-1992, were used and two comparable MRB score variables were derived. Logistic regression was used to explore the association between MRB and young adult SES. The moderating effect of three early life SES variables was assessed using logistic regression models with and without interaction parameters. Evidence to support the presence of moderation was determined by likelihood ratio tests $\mathrm{p} \leq 0.05$. Missing data were addressed using multiple imputation methods.

Results Adolescents had a median of two risk behaviours in BCS70 and three in ALSPAC. Adolescent MRB was negatively associated with young adult SES (university degree attainment) in BCS70 (OR 0.81, 95\% CI: 0.76, 0.86) and ALSPAC (OR $0.85,95 \%$ CI: $0.82,0.88)$. There was a dose response: each incremental risk behaviour resulted in reduced odds of university degree attainment. There was a negative association between MRB and occupational status at age 34 in BCS70 (adjusted OR 0.86 95\% CI: 0.82, 0.90). In BCS70, there was evidence that parental occupational status $(p=0.009)$, maternal education $(p=0.03)$ and household income $(p=0.03)$ moderated the effect of adolescent MRB on young adult SES with the negative effect of MRB on degree attainment being stronger for those from low socioeconomic backgrounds. There was no evidence in ALSPAC that early life SES moderated this relationship.

Conclusion Adolescence appears to be a critical time in the life course to address risk behaviours, due to the likelihood that behaviours established here may have effects in later in life. Intervening on adolescent MRB could improve adult SES outcomes and thus indirectly affect longer term health. Evidence for a moderation effect in the BCS70 but not ALSPAC highlights that alternative measures should be explored to capture the nuance of contemporary young adult SES.

\section{P33 SOCIOECONOMIC INEQUALITIES IN THE PREVALENCE AND MANAGEMENT OF HYPERTENSION: A MULTILEVEL ANALYSIS OF 13,605 MEN AND WOMEN IN CHILE}

\author{
${ }^{1,2} \mathrm{~A}$ Passi-Solar*, 'S Scholes, 'JS Mindell, ${ }^{1} \mathrm{M}$ Ruiz. ${ }^{1}$ Research Department of Epidemiology \\ and Public Health, University College London, London, UK; ${ }^{2}$ Departamento de Salud \\ Publica, Pontificia Universidad Catolica de Chile, Santiago, Chile \\ 10.1136/jech-2020-SSMabstracts. 127
}

Background Place-based characteristics have been implicated as determinants of socioeconomic inequalities in cardiovascularrelated health risk factors (such as hypertension) and in use of healthcare services. In Chile, one-third of adults are hypertensive. Chilean evidence has documented inequalities in hypertension by various measures of individual socioeconomic position (SEP). However, more research is needed to assess the contribution of area-level contextual factors such as income inequality on hypertension management inequalities. Methods Data came from the Chilean Health Surveys (2003, 2010, 2017: $\mathrm{N}=13,605$ participants aged 17 years and older). Our outcomes were hypertension and management indicators (e.g. treatment among hypertensives). Hypertension was defined as SBP/DBP of at least $140 / 90 \mathrm{mmHg}$ or use of antihypertensive medication. Years of formal education was our chosen measure of individual SEP; at the county-level, the Gini coefficient was used as an index of income inequality. Our models, adjusted for the complex survey design, were age (categorized as 17-64 or $65+$ years) -and gender-specific. We compared two models. First, a fixed model assessed inequalities using individual-level SEP. Secondly, multilevel analyses assessed inequalities using individual-level SEP after adjustment for the Gini coefficient. Inequalities were summarised using the Slope and Relative Indices of Inequality (SII and RII, respectively). For brevity, we report only the RII (values above 1 indicate higher outcomes amongst those with lower education).

Results Hypertension prevalence was 34.0\% (95\% CI: 31.6$36.4), 32.0 \%(29.9-34.2)$ and $30.8 \%(28.7-32.9)$ in 2003 , 2010 and 2017, respectively. Levels of treatment among hypertensives was $38.5 \%(34.9-42.3), 56.5 \%(52.3-60.6)$ and $65.2 \%(61.2-68.9)$ in 2003, 2010 and 2017, respectively. Inequalities in hypertension were higher in 2017 after adjustment for the Gini coefficient. For example, in the fixed-effects model, the RII for hypertension among persons aged below 
65 years was 2.9 (95\% CI: 1.6-5.2) and 5.8 (2.5-9.7) for males and females, respectively. After adjustment for the Gini coefficient, the RII increased to $4.2(2.6-6.6)$ and 6.3 (3.910.1) for males and females, respectively. Inequalities in treatment among older hypertensive females were similar in magnitude before and after adjustment for the Gini coefficient, with an estimated RII of $0.6(0.5-0.8)$ and $0.7 \quad(0.5-0.8)$ respectively.

Conclusion Multilevel analyses are required to better estimate inequalities. Chile currently needs interventions to improve the management of hypertension and simultaneously, decrease inequalities, first, in hypertension prevalence among younger males and females and, secondly, in its management among older hypertensive females.

The Chilean National Health Surveys 2003-2010-2017 were funded by the Chilean Ministry of Health.

This study is part of Alvaro Passi-Solar's PhD research, funded by the Chilean Ministry of Education (ANID-Conicyt).

\section{P34 SOCIOECONOMIC DISPARITIES IN THE DEVELOPMENT OF MULTIMORBIDITY IN SCOTLAND: THE BENEFITS OF APPLYING A LIFE COURSE LONGITUDINAL APPROACH}

${ }^{1} \mathrm{G}$ Cezard*, ${ }^{1} \mathrm{~K}$ Keenan, ${ }^{2} \mathrm{~F}$ Sullivan. 'School of Geography and Sustainable Development, University of St Andrews, St Andrews, UK; ${ }^{2}$ School of Medicine, University of St Andrews, St Andrews, UK

\subsection{6/jech-2020-SSMabstracts. 128}

Background There is widespread acknowledgement that we need to go beyond the single disease framework when trying to understand health inequalities across the life course. In Scotland, evidence based on primary care records showed that more than half of the people aged 65 years and older had at least two chronic diseases. Multimorbidity, defined as the cooccurrence of two or more chronic diseases, was also shown to be more prevalent in women and those with lower socioeconomic status (SES). The vast majority of multimorbidity research (and the indicators used) is situated within a crosssectional framework, and a typical goal is to identify disease clusters at a single point in time.

We aim to investigate the social patterning of multimorbidity trajectories in Scotland using both cross-sectional and longitudinal approaches.

Methods We use the Scottish Longitudinal Study (SLS) which links 1991, 2001 and 2011 censuses, vital events and other administrative or publicly available data sets for a 5\% representative sample of the Scottish population. Our subsample focuses on 120000 SLS individuals aged 40 years and over at the time of the Scottish census 2001, linked to their Scottish census 2011, hospitalisation and death records. Our sample is followed for up to 10 years (next census), censoring for death or emigration. Multimorbidity is identified using hospitalisation data and the list of comorbidities of the Charlson Index using Quan et al. algorithm (2005). Educational level in 2001 and the Scottish Index of Multiple Deprivation (SIMD) are used as SES proxies. Poisson and Cox regressions are used to explore SES inequalities in multimorbidity index at baseline and over time.

Results As expected, we find that multimorbidity is socially patterned: Individuals with lower education and who live in more deprived areas experienced faster levels of multimorbidity accumulation. In a separate analysis, we use single-channel sequence analysis to characterise multimorbidity trajectories and sequences of disease onset and relate this to social inequalities.

Conclusion This study highlights the need to go beyond a cross-sectional approach in researching multimorbidity differences. It shows the influence of the timing of and sequencing of disease onset in shaping social health inequalities in later life and it also provides a characterisation of multimorbidity progression through the application of longitudinal methods used in life course studies. With new data linkages, capturing detailed disease onset, and timing linked with social factors, research of the social patterning of multimorbidity progression can be advanced and fed into prevention strategies.

\section{P35 AN INDIVIDUAL PARTICIPANT DATA META-ANALYSIS EXAMINING HEALTH INEQUALITIES FACING OLDER LESBIAN, GAY AND BISEXUAL PEOPLE AGED 50 AND OVER IN THE UNITED KINGDOM}

D Kneale*. EPPI-Centre, University College London, London, UK

\subsection{6/jech-2020-SSMabstracts.129}

Background Older lesbian, gay and bisexual (LGB) people were born at a time same sex activity between men was a criminal offence, and where social and legislative conditions permitted discrimination against sexual minorities across a spectrum of domains. Minority Stress Theory posits that LGBT people are at an elevated risk for poorer health because of their exposure to social stress related to prejudice and stigma. Modelling the health and care trajectories of lesbian, gay and bisexual (LGB) is essential to identify inequalities and support needs, yet because of the small sample of LGB people in any one survey, current evidence relies on studies that have poor generalisability and low power. This study assesses the magnitude of health and care inequalities among older LGB people across ten outcomes, informed by evidence on the health trajectories and distinct history of LGB people in the UK.

Methods A systematic review was conducted of representative data sources on older LGB and heterosexual people's health and care status in the UK. Individual Participant Data (IPD) meta-analysis was employed to synthesise data. To account for the intricacies of individual datasets, the analysis employed a two-stage approach where an odds ratio (OR) and standard error was calculated for each dataset individually, before being meta-analysed through DerSimonian and Laird random effects models.

Results Our largest model incorporated data from 25 different datasets and provided an unparalleled sample size (over 2,500 LGB men and women) to measure the magnitude of sexualitybased health inequalities in later life. We find that among men aged 50 and over, being gay, bisexual or having another nonheterosexual orientation is associated with an increased risk of reporting a long-term illness and limitations due to health or illness. Indicators of mental health also suggest that gay and bisexual men are more likely to report low life satisfaction and substantially more likely to have attempted suicide over their life time (Adjusted Odds Ratio: 2.29; 95\% Confidence Interval: 1.19-4.42). We find that among women, differences in health are apparent with regards to self-rated health as well as with engagement with risky health behaviours with higher levels of smoking and frequent alcohol consumption. 